\title{
Modeling of Spatial Solitons in Twisted Nematics Waveguides
}

\author{
M.A. Karpierz**, K.A. Brzdąkiewicz and Q.V. Nguyen \\ Faculty of Physics, Warsaw University of Technology \\ Koszykowa 75, 00-662 Warsaw, Poland
}

\begin{abstract}
Theoretical analysis of light beam propagation in twisted nematic liquid crystalline waveguides is presented. Due to the optical reorientation nonlinearity, the light beam changes the direction of propagation and optical spatial soliton is formed. Modeling of such behavior is presented by using $(2+1)$-dimensional vector beam propagation method as well as simplified (1+1)-dimensional method. Although both approaches give similar results, the differences are discussed in detail.
\end{abstract}

PACS numbers: 42.65.Wi, 42.70.Df

\section{Introduction}

Nematic liquid crystals are interesting media for application in nonlinear optics with nonlinear phenomena arising mainly from molecular reorientation or/and thermal effects [1, 2]. In particular, nematic liquid crystals are suitable for creation of spatial optical solitons. The reorientation nonlinearity in nematic liquid crystals can govern spatial solitons in both waveguide and bulk geometry. Such solitons require low power of light and they can be controlled by the state of light polarization or an external electric field. Experimental results demonstrated that light beams of only milliwatts could be self-trapped at distances of a few millimeters [3].

Solitons in nematic liquid crystals were analyzed in layer structures with the planar or homeotropic texture $[4,5]$ and in capillaries with axial orientation [6-8]. In this paper twisted nematics configuration is analyzed. Light beam propagating in such medium diffracts and due to the structural anisotropy propagates at some angle to the direction of the input light $[9,10]$. Optical nonlinearity causes that for higher light intensities the light beam is self-focused and additionally changes the direction of propagation.

*corresponding author; e-mail: karpierz@if.pw.edu.pl 


\section{Propagation of light beam}

The light beam propagating in a layer filled with twisted nematic liquid crystal (see Fig. 1) is taken into consideration. The electromagnetic field with dominating $E_{y}$ component of the electric field is assumed. In nonlinear case the liquid crystal molecules are forced to reorient in the $y z$ plane. This case is described by electrical permittivity tensor

$$
\varepsilon=\left(\begin{array}{ccc}
\varepsilon_{\perp} & 0 & 0 \\
0 & \varepsilon_{\perp}+\Delta \varepsilon \cos ^{2} \theta & \Delta \varepsilon \sin \theta \cos \theta \\
0 & \Delta \varepsilon \sin \theta \cos \theta & \varepsilon_{\perp}+\Delta \varepsilon \sin ^{2} \theta
\end{array}\right)
$$

where $\Delta \varepsilon=\varepsilon_{\|}-\varepsilon_{\perp}$ is an optical anisotropy, $\varepsilon_{\perp}=n_{\mathrm{o}}^{2}$ is an ordinary and $\varepsilon_{\|}=n_{\mathrm{e}}^{2}$ is an extraordinary electric permittivity, and $\theta$ is an orientation angle measured as an angle between the liquid crystal molecules axis and the $y$-axis.
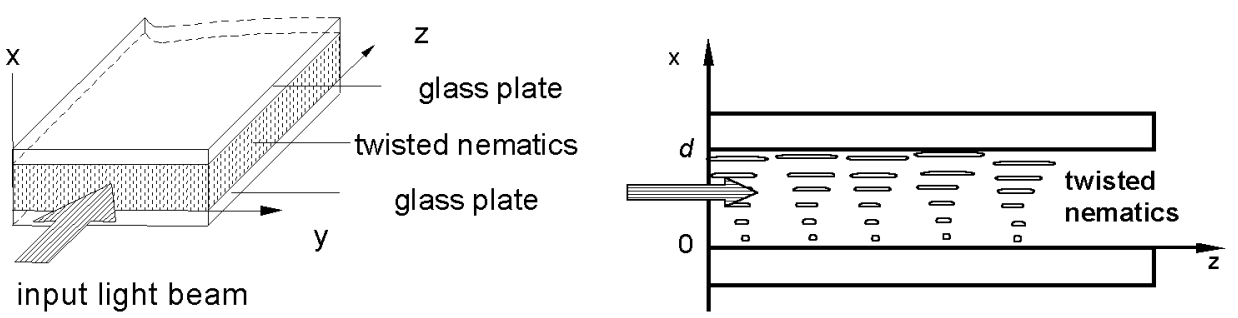

Fig. 1. Schematic drawing of planar waveguides with twisted nematic liquid crystal.

The distribution of the orientation angle $\theta$ is calculated from the EulerLagrange equation in the form considered for the twisted nematic liquid crystals [2]:

$$
\frac{\mathrm{d}^{2} \theta}{\mathrm{d} x^{2}}+\frac{\mathrm{d}^{2} \theta}{\mathrm{d} y^{2}}+\frac{\varepsilon_{0} \Delta \varepsilon}{4 K_{22}}\left[2 E_{y} E_{z} \cos 2 \theta-\left(\left|E_{y}\right|^{2}-\left|E_{z}\right|^{2}\right) \sin 2 \theta\right]=0
$$

where $K_{22}$ is an elastic constant corresponding to the twist deformation. In the absence of the electrical field, the orientation angle in twisted nematics is a linear function of $x$ coordinate: $\theta(x)=\theta(0)+\Delta \theta x / d$, where $\Delta \theta \leq \pi / 2$. In this paper the calculations are done for boundary conditions equal to $\theta(0)=0$ and $\theta(d)=\pi / 2$ which corresponds to the initial orientation $\theta(x)=\pi x / 2 d$.

Propagation of light beam in twisted nematics was investigated by using the numerical beam propagation method with the TE-polarized Gaussian beam in the input plane. Numerical results were calculated for liquid crystal layer with refractive indices $n_{\mathrm{o}}=1.52$ and $n_{\mathrm{e}}=1.69$ surrounded by glass plates with refractive index $n_{\mathrm{c}}=1.45$, with the thickness $d=10 \mu \mathrm{m}$ and for the wavelength $\lambda=842 \mathrm{~nm}$.

Numerical results in Fig. 2 present the dependence of $\int\left|E_{y}\right|^{2} \mathrm{~d} x$ of the light beam propagating in the analyzed structure. Calculations were done by numerical integration of Maxwell equations (with three components of electrical field) with 


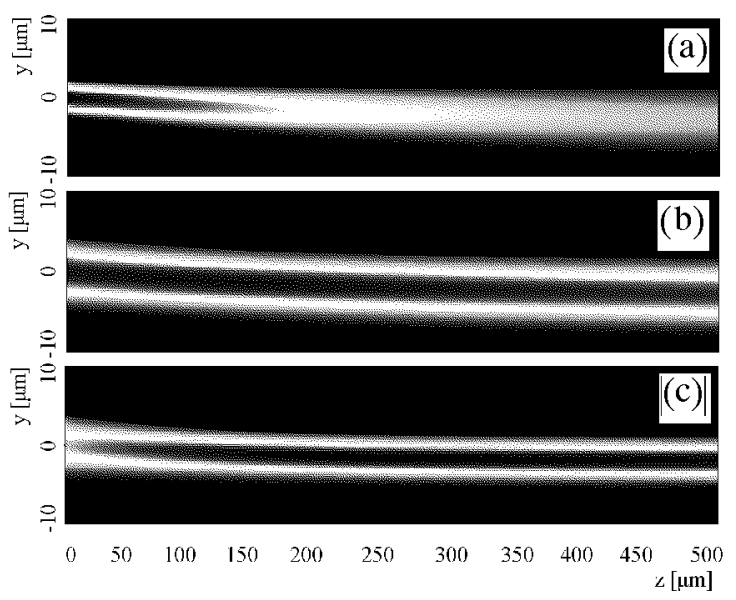

Fig. 2. Dependence of $\int\left|E_{y}\right|^{2} \mathrm{~d} x$ in the light beam propagating in twisted nematics waveguide (a) for the linear case, i.e. for the light power $P \rightarrow 0$, and in the presence of nonlinear reorientation: (b) for the power $P=150 \mathrm{~mW}$, and (c) $P=700 \mathrm{~mW}$.

the electrical permittivity described by Eqs. (1), (2). In the linear case (for low light power) the typical diffracted light beam propagating at some angle to the $z$-axis exists (Fig. 2a). For larger light power the diffraction is compensated by the nonlinearity and soliton-like beam propagating at the direction not perpendicular to the $z$-axis is obtained (Fig. 2b). Next, for large enough value of the light power the beam forms spatial soliton propagating parallel to the $z$-axis (Fig. 2c).

\section{3. $(1+1)$-dimensional approximation}

Results presented in Fig. 2 were obtained by application of $(2+1)$-dimensional beam propagation method with full vector electromagnetic fields, i.e. Maxwell equations with three components of the electric field were solved. However, the planar waveguide structure allows to reduce number of equations needed to be solved by assuming that the dependence on $x$ is the same as in the waveguide mode. Assuming that the $E_{z}$ component of the field is much weaker than the $E_{y}$ component, $E_{z} \ll E_{y}$, the electric fields can be introduced in the form [10]:

$$
\begin{aligned}
& E_{y}=A(y, z) \varphi(x) \exp \left(\mathrm{i} \omega t-\mathrm{i} k_{0} N z\right), \\
& E_{z}=A(y, z) \phi(x) \exp \left(\mathrm{i} \omega t-\mathrm{i} k_{0} N z\right),
\end{aligned}
$$

where $\varphi(x) \exp \left(\mathrm{i} \omega t-\mathrm{i} k_{0} N z\right)$ and $\phi(x) \exp \left(\mathrm{i} \omega t-\mathrm{i} k_{0} N z\right)$ are components of the planar waveguide mode, $N$ is an effective refractive index and $A$ is a complex amplitude slowly varying with respect to $z$. The mode envelopes $\varphi(x)$ and $\phi(x)$ fulfill the equations for an electric permittivity corresponding to the initial orientation 
of the nematics. Consequently, the slowly varying complex amplitude $A$ fulfills the $(1+1)$-dimensional equation

$$
2 \mathrm{i} k_{0} N \frac{\partial}{\partial z} A=\frac{\partial^{2}}{\partial y^{2}}\left(1+\kappa_{1}\right) A-\mathrm{i} k_{0} N \frac{\partial}{\partial y} \kappa_{2} A+k_{0}^{2} N^{2} \kappa_{3} A
$$

The nonlinear coefficients $\kappa_{p 0}(p=1,2,3)$ could be approximated by analytical formulae $[9,10]$ :

$$
\begin{aligned}
& \kappa_{1}=\kappa_{10}+\alpha_{10} \frac{|A|^{2}}{1+\left|A / A_{1 \mathrm{~S}}\right|^{2}}, \\
& \kappa_{2}=\kappa_{20}-\alpha_{20} \frac{|A|^{2}}{1+\left|A / A_{2 \mathrm{~S}}\right|^{2}}, \\
& \kappa_{3}=\alpha_{30} \frac{|A|^{2}}{1+\left|A / A_{3 \mathrm{~S}}\right|^{2}},
\end{aligned}
$$

where saturation amplitudes $A_{\mathrm{S}}$ as well as values of $\kappa_{p 0}$ and $\alpha_{p 0}(p=1,2,3)$ could be fitted from exact calculations.

All coefficients depend on light intensity in a saturated-like form and all are much lower than $1\left(\kappa_{p} \ll 1\right)$. The coefficient $\kappa_{2}$ is responsible for walking off the light beam. For low intensities (in the linear case: $A \rightarrow 0) \kappa_{2}$ is the largest (equal to $\left.\kappa_{20}\right)$ and increasing intensities causes decreasing their values $\left(\kappa_{2} \rightarrow 0\right.$ for $\left.A \rightarrow \infty\right)$. It means that for higher intensities the walk-off of the light beam is lower, i.e. the light beam direction is changing with changing the light power. The last term in Eq. (5), connected with the coefficient $\kappa_{3}$, is purely nonlinear and it is responsible for self-focusing of light beam and creation of the spatial soliton. However, this nonlinear component could also modify the direction of light beam propagation.

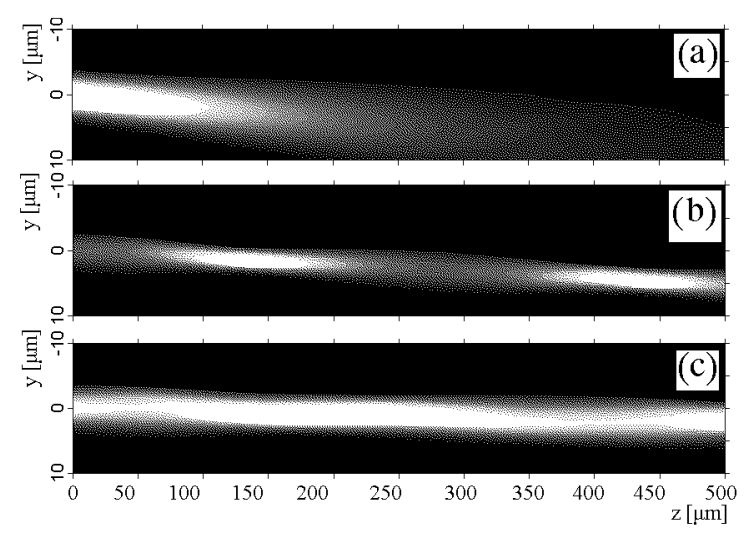

Fig. 3. Dependence of $|A|^{2} \propto \int\left|E_{y}\right|^{2} \mathrm{~d} x$ in the light beam calculated in (1+1)-dimensional approximation: (a) in linear case $P \rightarrow 0$, (b) for the power $P=$ $150 \mathrm{~mW}$, and (c) $P=700 \mathrm{~mW}$. 
The numerical results are presented in Fig. 3, where the contours of $|A|^{2}$ are plotted. The values of parameters approximating the nonlinear coefficients $\kappa$ were fitted as follows: $\kappa_{20}=0.07, \alpha_{20}=0.025,\left(A_{2 \mathrm{~S}}\right)^{2}=3.6, \alpha_{30}=0.06,\left(A_{3 \mathrm{~S}}\right)^{2}=1.4$, and $\kappa_{1}=0$ were taken. The input light beam with the same Gaussian shape as in Fig. 2 was used. Obtained behavior is similar to those from Fig. 2: due to the nonlinearity light beam is self-focused and changes the direction of propagation. However, in comparison to the more exact result presented in Fig. 2, the simplified approach leads to oscillation of light beam width and changing the direction of propagation requires higher power.

The main source of differences between exact and simplified results is an assumption done in Eqs. (3), (4) that the electric field profiles $\varphi(x)$ and $\phi(x)$ do not depend on the light intensity. This assumption is valid in a low-nonlinearity limit which is not sufficiently satisfied for analyzed waveguide. The electric field profiles across the waveguide obtained in (2+1)-dimensional calculations are presented in Fig. 4. The profiles in the center of the light beam width of the power $P=700 \mathrm{~mW}$
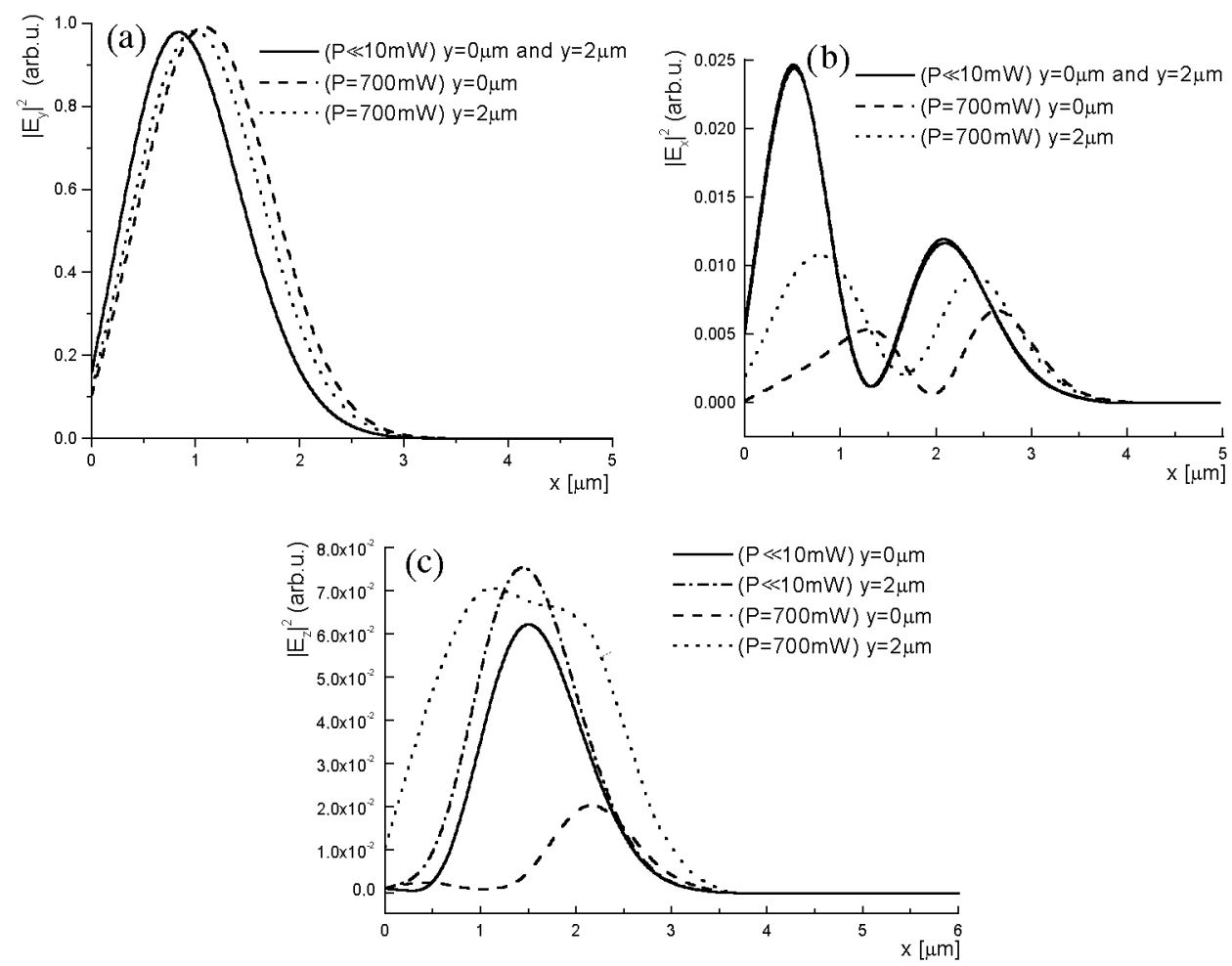

Fig. 4. Distribution of the electric field components across the waveguide in the center of the light beam and $2 \mu \mathrm{m}$ far from the center in the linear case (solid and dash-dotted lines) and for the light power $700 \mathrm{~mW}$ (dash and dotted lines): (a) $\left|E_{y}\right|^{2}$, (b) $\left|E_{x}\right|^{2}$, (c) $\left|E_{z}\right|^{2}$. 


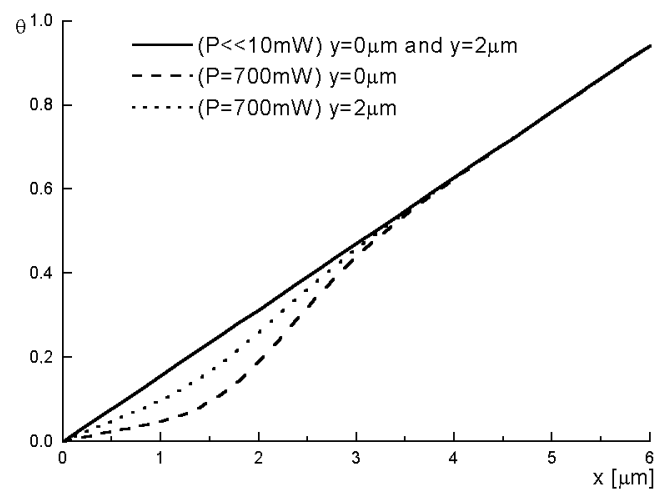

Fig. 5. Distribution of the orientation angle $\theta$ across the wa veguide for the linear case (solid line) and for the light power $700 \mathrm{~mW}$ in the center (dashed line) and $2 \mu \mathrm{m}$ far from the center of light beam (dotted line).

(dashed lines) differ from the linear profiles (solid line) as well as from the profiles at the light beam edge ( $2 \mu \mathrm{m}$ far from the center), where the light intensity is lower (dotted line). Consequently, the nonlinear reorientation of the nematic liquid crystal is different in the center than at the beam edge (see Fig. 5). Let us note that profile of the $E_{z}$ component is also different at the light beam edge in the linear case (dash-dotted line in Fig. 4c), which is caused by the diffraction of the light beam.

\section{Conclusions}

Presented numerical results show the light beam self-trapping and changing the direction of propagation in the twisted nematics waveguides due to the reorientation nonlinearity. Theoretical model assuming low-nonlinearity leads to the $(1+1)$-dimensional equation which is much simpler and faster in calculations than the full vector $(2+1)$-dimensional method. Numerical results obtained in the framework of the simplified model are qualitatively comparable with the exact calculations. Simplified model also partially takes into consideration the nonlocality of the reorientation nonlinearity, because it is included in the method of calculation of the nonlinear coefficients $\kappa_{p}$. However, in the simplified model some effects appear, like oscillation of the beam width, which do not exist in the results of exact calculations.

Changing the direction and self-focusing of light beam is caused by the nonlinear reorientation of the liquid crystal. Reorientation increases the effective refractive index and decreases the structural anisotropy by reducing the region with twisted orientation. In presented calculations both effects (self-focusing and changing the direction of propagation) require relatively large light power. However, optimization of the waveguide thickness and the size of the light beam should reduce 
necessary value of the light power. The proposed configuration of liquid crystalline waveguide can be applied to switching of the light beam in the low-power all-optical systems.

\section{References}

[1] I.C. Khoo, S.T. Wu, Optics and Nonlinear Optics of Liquid Crystals, World Scientific, Singapore 1993.

[2] N.V. Tabiryan, A.V. Sukhov, B.Ya. Zel'dovich, Mol. Cryst. Liq. Cryst. 136, 1 (1986).

[3] M.A. Karpierz, in: Soliton-Driven Photonics, Eds. A.D. Boardman, A.P. Sukhorukov, Kluwer, Dordrecht 2001, p. 41.

[4] M.A. Karpierz, M. Sierakowski, M. Świłło, T.R. Woliński, Mol. Cryst. Liq. Cryst. 320, 157 (1998); M.A. Karpierz, Phys. Rev. E 66, 036603 (2002).

[5] M. Peccianti, A. De Rossi, G. Assanto, A. De Luca, C. Umeton, I.C. Khoo, Appl. Phys. Lett. 77, 7 (2000); M. Peccianti, G. Assanto, Opt. Lett. 26, 1690 (2001); 26, 1791 (2001); Phys. Rev. E 65, 035603 (2002).

[6] E. Braun, L.P. Faucheux, A. Libchaber, Phys. Rev. A 48, 611 (1993).

[7] D.W. McLaughlin, D. J. Muraki, M.J. Shelley, X. Wang, Physica D 88, 55 (1995).

[8] M. Warenghem, J.F. Henninot, G. Abbate, Mol. Cryst. Liq. Cryst. 320, 207 (1998); F. Derrien, J.F. Henninot, M. Warenghem, G. Abbate, J. Opt. A, Pure Appl. Opt. 2, 332 (2000).

[9] M.A. Karpierz, Acta Phys. Pol. A 99, 161 (2001).

[10] M.A. Karpierz, M. Sierakowski, T.R. Woliński, Mol. Cryst. Liq. Cryst. 375, 313 (2002). 